\title{
Study of the Selection Criteria for the Auto-Chemical Analyser in the Medical Instruction below Hospital Level based AHP
}

\author{
Do-Hyun Kim¹, Won-Chul Seo², Jong-Seok Seo ${ }^{3}$, Young-Seok Ock ${ }^{3}$ \\ ${ }^{1} \mathrm{CS}$ Team/BioSystems Korea \\ 150 JoJung-daero Hanam-city, Kyoung gi-do, Korea \\ dhkim@biosystems.kr \\ ${ }^{2}$ Division of Systems Management and Engineering/Pukyong National University \\ 45 Yongso-ro Nam-gu, Busan, Korea \\ wcseo@pknu.ac.kr \\ ${ }^{3}$ Graduate School of Management of Technology/Pukyong National University \\ 365 Sinseon-ro Nam-gu, Busan, Korea \\ bellstones@naver.com; ysock@pknu.ac.kr
}

\section{Extended Abstract}

In order to solve the patient biased phenomenon of large hospitals, the need strengthening the medical institutions below hospital level has been raised [1]. The clinical chemistry tests for the Medical Institutions below hospital level consist of manual, kit, and auto-method. The auto-chemical analyzer is preferred because of the precision, accuracy, and efficiency [2]. In this study, the selection criteria for the auto-chemical analyzer are investigated and by using AHP method ([3], [12]), the priority of user's consideration at the medical institutions below hospital level is figured out. The implementation of AHP method is as follows.

Step 1: Model the problems as hierarchy

Step 2: Evaluate the hierarchy by paired comparisons

Step 3: Establish priorities

Step 4: Check the consistency of the judgments

The goal is choosing the auto-chemical analyzer. The group of options for reaching the goal is composed of the analyzer, the reagent, the service, the agency and the reputation. There are 19 selection criteria - the analyzer (5), the reagent (3), the service (4), the agent (4) and the reputation (3), Figure 1. Of the 19 selection criteria, stability of analyzer, stability of reagent, response manners and rapid response to customer's complaint were highly important. They are directly related to user's manipulation of the device.

To quantify an abstract idea about the auto-chemical analyzer selection factors of medical laboratory scientist in the medical Institutions below hospital level, the priority was clarified. So the result of this study will be used as basic tools for the competition strategy of the medical device company.

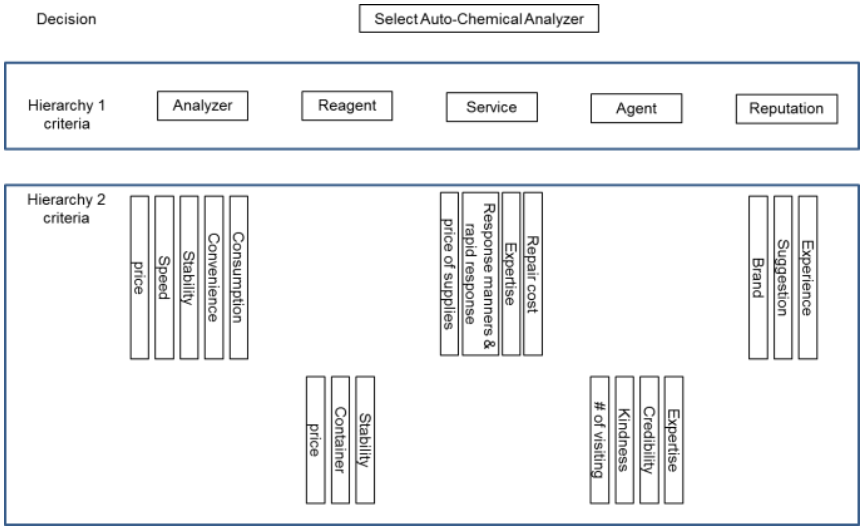

Fig. 1: AHP Hierarchical Structure Model for Auto-Chemical Analyzer. 


\section{References}

[1] K. H. Ko, "Medical Services Industry Trends and Policy Research," Korea Health Industry Development Institute, vol. 12, 2013.

[2] J.-D. Yeo, "A study on the Effect of Medical Device Purchase Decision Making," Healthcare Management Consulting, vol. 2, no. 1, 2008.

[3] C. S. Park, "Credit Evaluation Model for Medical Venture Business by the Analytic Hierarchy Process," The Korean Society of Business Venturing, vol. 6, no. 2, pp. 133-147.

[4] M. Choi, "An AHP Study on Priority Factors Affecting Healthcare Consumers' Selection of Hospitals," Korea Advertising Society, vol. 89, 2011. 